\title{
Performance assessment of fragmentation mechanisms for vehicular delay-tolerant networks
}

\author{
João AFF Dias ${ }^{1}$, Joel JPC Rodrigues ${ }^{1 *}$, João N Isento ${ }^{1}$, Paulo RBA Pereira ${ }^{2}$ and Jaime Lloret $^{3}$
}

\begin{abstract}
Vehicular Delay-Tolerant Networks (VDTNs) are a new approach for vehicular communications where vehicles cooperate with each other, acting as the communication infrastructure, to provide low-cost asynchronous opportunistic communications. These communication technologies assume variable delays and bandwidth constraints characterized by a non-transmission control protocol/internet protocol architecture but interacting with it at the edge of the network. VDTNs are based on the principle of asynchronous communications, bundle-oriented communication from the DTN architecture, employing a store-carry-and-forward routing paradigm. In this sense, VDTNs should use the tight network resources optimizing each opportunistic contact among nodes. Given the limited contact times among nodes, fragmentation appears as a possible solution to improve the overall network performance, increasing the bundle delivery probability. This article proposes the use of several fragmentation approaches (proactive, source, reactive, and toilet paper) for VDTNs. They are discussed and evaluated through a laboratory testbed. Reactive and toilet paper approaches present the best results. It was also shown that only the source fragmentation approach presents worst results when compared with non-fragmentation approaches.
\end{abstract}

Keywords: vehicular delay-tolerant networks, vehicular communications, fragmentation, performance evaluation, prototype

\section{Introduction}

Over the past years, researchers and the automotive industry have driven joint efforts concerning Inter-Vehicle Communication in order to provide better Intelligent Transportation Systems, as well as drivers and passenger's assistance services [1-3]. The potential applications of these networks that include, but is not limited to, road traffic optimization, road safety, monitoring, driving assistance, and a wide variety of commercial and entertainment applications, also contribute to the growing interest of vehicular networks. Comparing to other communication systems, vehicular networks are characterized by several and unique features, namely the intermittent connectivity and the potential non-existence of an end-to-end path [4]. However, this type of networks has to deal with challenging issues [5] (e.g., the high mobility of vehicles, the constant network topology changing, or even the network scale). To overcome the above-mentioned issues, several approaches are emerging, such as Vehicular Ad Hoc

\footnotetext{
* Correspondence: joeljr@ieee.org

${ }^{1}$ Instituto de Telecomunicações, University of Beira Interior, Covilhã, Portugal Full list of author information is available at the end of the article
}

Networks (VANETs) [6,7], Delay-Tolerant Networks (DTNs) [8], and more recently, Vehicular Delay-Tolerant Networks (VDTNs) [9] that are considered in this study.

VDTNs use mobile nodes to enable communications in remote and sparse scenarios characterized by disconnection, as well as urban scenarios. Three different types of nodes may be combined in VDTNs networks: terminal, relay, and mobile nodes. Terminal nodes are considered access points to the VDTN network. Usually, they are placed at the edge of the network. Stationary relay nodes are fixed devices placed at roads intersections for increasing the network connectivity, allowing mobile nodes to put and gather data. These relay nodes increase the number of contacts among mobile nodes in order to increase the bundle delivery probability by decreasing the bundles average delay [10]. Finally, mobile nodes move along roads, carrying data between terminal nodes. These mobile nodes may also generate and receive data, acting as terminal nodes too.

Although taking several contributions from DTNs, the VDTN architecture differs from them by introducing an IP over VDTN approach. VDTNs also perform an 
out-of-band signalling, allowing the separation between the control and data planes [9]. The control plane assumes the signalling and resource reservation functionalities in order to schedule data exchange among nodes. It executes signalling messages exchange, resources reservation to be used in data plane and routing functionalities, among others. The data plane includes data carrying and exchange, queuing and scheduling, and traffic classification. Figure 1 illustrates control and data planes operation.

VDTNs implement a store-carry-and-forward paradigm in order to solve problems caused by disconnection and intermittency. Figure 2 presents the interactions between VDTN network nodes and the store-carry-and-forward paradigm.

Even with some distinctions when compared with the DTN approach, VDTNs have to deal with the same problems related to the network connectivity. Most of them are due to the high mobility and velocity of vehicles. These aspects will directly affect the network performance by causing constant network topology changes and limit the contacts' duration. The contact duration is a key feature in the study of fragmentation mechanisms for VDTNs. When two network nodes meet and establish contact, they have a limited period to exchange data bundles. If the contact is suddenly interrupted and there are still bundles being exchanged, these bundles will be incomplete. If no fragmentation mechanism is considered, the incomplete bundles will be discarded resulting in a waste of network resources. This waste may lead to an increase of the bundle average delay and, consequently, decreases the bundle delivery probability. Thus, in order to maximize the use of tight network resources and increase the delivery ratio, this study presents, discusses and analyses the performance of several fragmentation mechanisms for VDTNs. The following fragmentation approaches are studied: proactive, source, reactive and toilet paper.

The rest of the article is organized as follows. A brief overview focusing on fragmentation for vehicular communications is presented in Section 2, while Section 3 presents and describes the design of the proposed fragmentation mechanisms for VDTNs. Section 4 presents the laboratory testbed used for performance evaluation studies, and Section 5 focuses on the discussion of the obtained results. Finally, Section 6 concludes the article and points some directions that will be considered for future study.

\section{Related study}

This section elaborates on the state-of-the-art about fragmentation, considering different approaches, ranging from IP to vehicular networks, and the corresponding approaches to overcome it. In IP networks, fragmentation happens when an IP datagram has to travel through a network with a Maximum Transmission Unit (MTU)

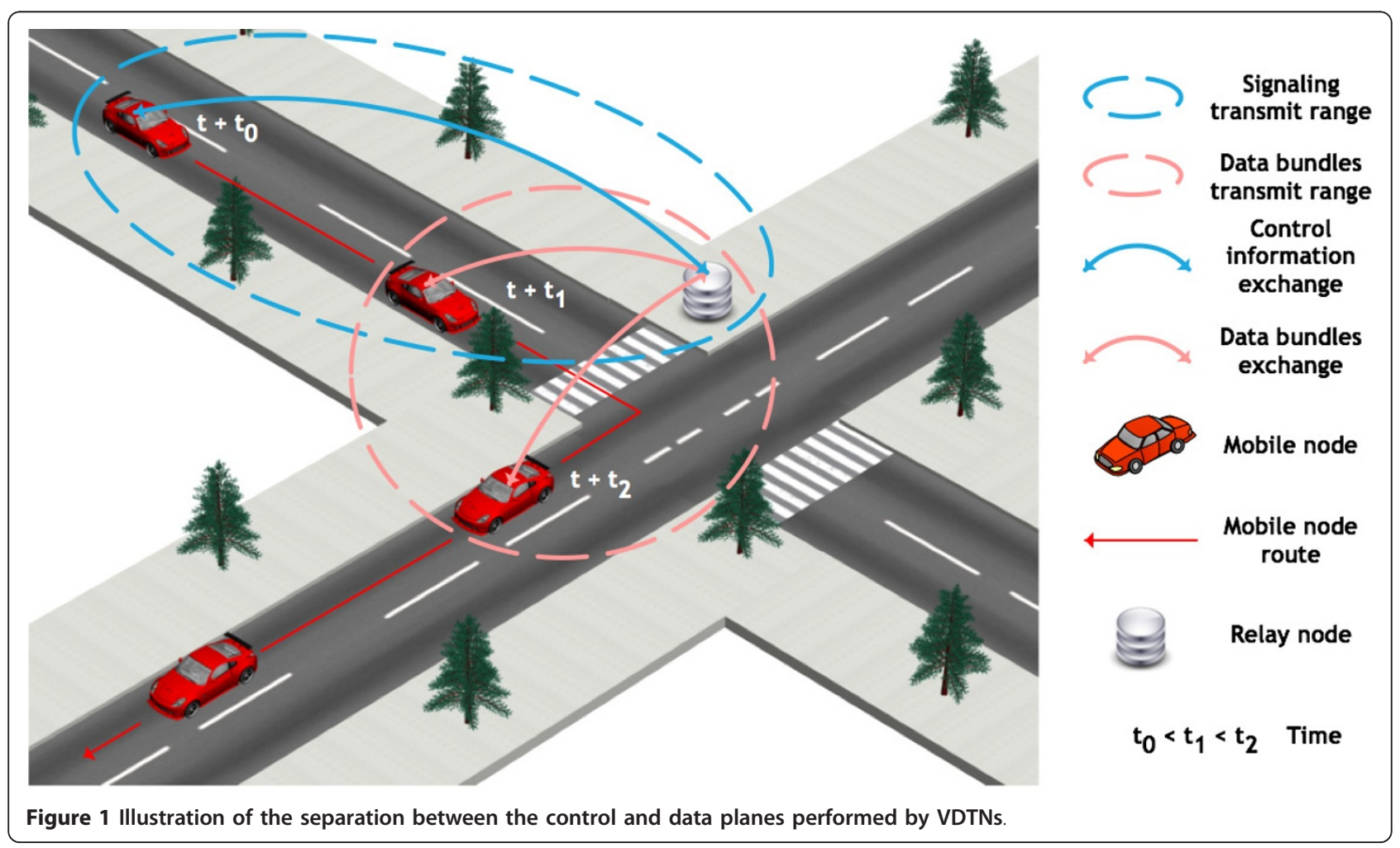




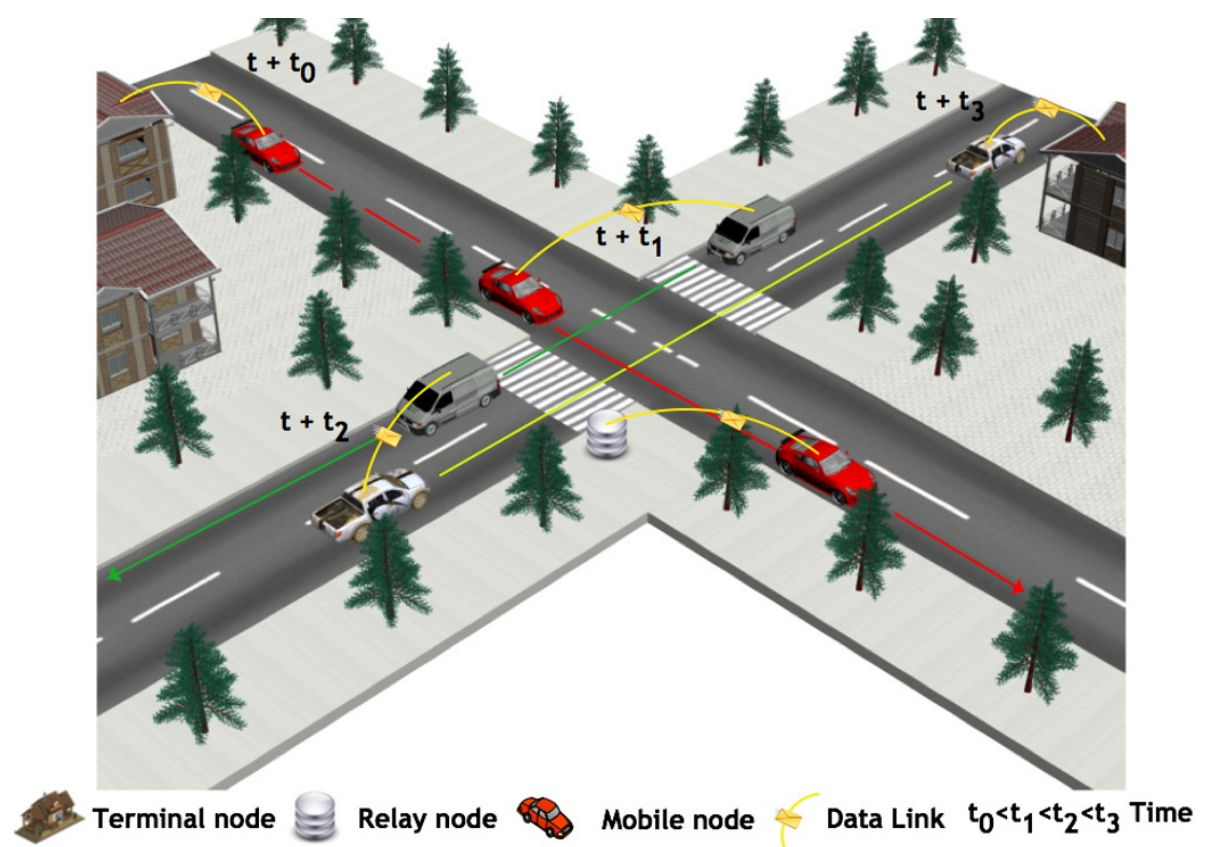

Figure 2 Illustration of the store-carry-and-forward paradigm for VDTNs and the interactions between the three types of VDTN network nodes (terminal, relay, and mobiles nodes).

that is smaller than the size of the IP datagram. The IP datagram is divided into fragments that will be reassembled later at the receiving host. The reassembly process uses several fields of the IP header such as IP Source, Destination, Identification, Total Length and Fragment Offset, or even the flags "More Fragments" and "Don't Fragment". The mechanisms for IP fragmentation and reassembly are reported in [11].

Fragment datagrams in an IP network may lead to a performance loss or to communications failures that may increase the probability of packet loss [12]. For these reasons, the IP fragmentation, despite being allowed, is considered harmful. A possible solution to avoid fragmentation is to determine the MTU of a certain path, in a dynamic way by sending multiple packets with different sizes with the IP header "don't fragment" flag active. Other solutions may consider the choice of the MTU based on the applications demands and conventional prospects on the operating environment.

In vehicular networks, fragmentation is processed differently than IP fragmentation. This happens because, in vehicular networks, there is an absence of an end-to-end path, resulting in an intermittent connectivity. This intermittent connectivity requires that every contact opportunity must be used in the best possible way in order to exchange as much bundles as possible. The main problem of contact opportunities in vehicular networks is their limited time. This represents an important limitation, and motivates the use of fragmentation approaches in such networks. Fragmentation may also occur due to buffer space constraints. When an entire bundle cannot be exchanged because a node does not have enough space to receive it, this bundle should be fragmented.

The authors of [13] propose a rate-adaptive protocol that allows dynamic fragmentation in wireless local area networks. This protocol tries to improve the throughput based on fragment transmission bursts and channel information. The amount of data for the next transmission is selected based on the channel information from previous transmissions.

Ginzboorg et al. [14] formalize message fragmentation in disruptive networks, such as vehicular networks, and investigate the impact of fragmentation on message forwarding over a single link. Authors also discuss several fragmentation strategies for source nodes.

Legner [15] enumerates several approaches to overcome fragmentation in mobile ad hoc networks, identifying the modification of the node trajectory [16], Epidemic routing approach [17] and delaying the message relay [18].

In [19], an adaptive fragmentation scheme for VANETs is presented. This approach relies on wireless channel time varying property and on the VANETs networks load conditions.

Although there are several approaches to handle fragmentation in VANETs, some authors prefer to study strategies to overcome the fragmentation problem. Joshi [20] proposes a geocast protocol that implements a 
mechanism to overcome network fragmentation and improve the performance of networks with sparse vehicle distribution. In this study, three main strategies to overcome fragmentation were identified: new neighbor approach [18], periodic retransmissions [21] and vehicle message ferry.

DTNs consider two types of fragmentation strategies: proactive and reactive. In the proactive approach, nodes are able to divide bundles into multiple fragments. These resulting fragments will be transmitted and processed as independent bundles. Only the final destination has the ability to reassemble all the fragments into the original bundle. This strategy is called proactive fragmentation because nodes perform the fragmentation process based on previous information about buffer conditions of the next hop and available link time. Only after, the fragmentation processed fragments are transmitted. The proactive approach considers a special case, called source fragmentation [22]. In this approach, bundles are fragmented at the time of its generation. After that, no more fragmentation is allowed. In order to perform such approach, source nodes split bundles in $n$ non-overlapping fragments with similar size. Thus, these fragments are forwarded sequentially.

In the reactive strategy, and unlike the proactive strategy, bundles are fragmented during the bundle transmission when the available link suffers from an unexpected failure. When this happens, both intervenient nodes have to determine which part of the bundle was successfully transferred and which part was not. Thus, the receiver node creates a bundle with the successfully received part of the bundle while the sender creates a fragment with the remaining part of the bundle. The toilet paper [23] approach is a variant of the reactive fragmentation strategy, where the size of the resulting fragment is not arbitrary, but defined by the originator.

The approach taken into account for DTNs may be extended to the VDTN architecture, since they share several features. The study proposed in [24] confirms this statement. It presents several fragmentation techniques for VDTNs in order to optimize the efficiency of data delivery for the case of the short node contacts that characterize vehicle networks. Another study considering VDTNs is presented in [25]. In this study, the authors explore the use of node localization in vehicles, offered by the global positioning system (GPS). The use of GPS allows the estimation of contacts duration that will prevent the transmission of incomplete bundles. With this information, vehicles only schedule the transmission of bundles that will be completely transmitted. Although permitted, this study does not contemplate any fragmentation mechanism. This section overviewed the most relevant literature about fragmentation mechanism. Some of them can be adapted and applied to VDTNs. It is expected that fragmentation strategies improve the VDTN network performance.

\section{Fragmentation strategies for VDTNs}

VDTNs make use of vehicles to carry data between network nodes. The high mobility of these vehicles leads to constant network changes and limited contact durations. When two vehicles meet, they start to exchange signaling messages in order to determine the next hop conditions (e.g. buffer space and power status) to perform the routing decisions and select which bundles should be exchanged among them. This process is performed at the control plane. Afterwards, if there are bundles to exchange, nodes start the data transfer among them (performed at the data plane). This process is performed until the contact time expires or the connection is broken. If such occurs, and a bundle is still being transferred, the receiver node will have an incomplete bundle. Usually, this incomplete bundle will be deleted resulting in a waste of network resources. If no fragmentation mechanisms are considered, then the bundle has to be retransmitted. This will contribute to an increase of the bundle delivery delay and consequently to an increase of the probability of this bundle to be dropped due to its Time-to-Live (TTL) expiration.

To improve the overall performance of VDTNs by increasing the bundle delivery ratio and decreasing the bundle delivery delay, several fragmentation strategies based on the DTN architecture are proposed (proactive, proactive source, reactive and toilet paper).

\subsection{Proactive fragmentation}

Proactive fragmentation may be performed at any network node, when the amount of data to exchange is higher than the allowed time for a contact. This type of fragmentation is performed when the VDTN node is performing the control plane operations. At each contact opportunity, both nodes in contact perform control plane functions to identify the contact time and select which bundles should be transferred. Afterwards, based on knowledge about the expected uptime of the available link or the buffer status of the next hop, the fragmentation module determines which bundles will be entirely transferred and which bundles may be fragmented. After the bundle fragmentation, fragments will be treated as independent bundles for routing decisions and buffer management. Only the final destination of fragments has the ability to reassemble all the fragments into the original bundle. If a fragment does not reach the destination, due to TTL expiration or to buffer congestion, the bundle is lost. Figure 3 illustrates the proactive fragmentation module operations described above. 


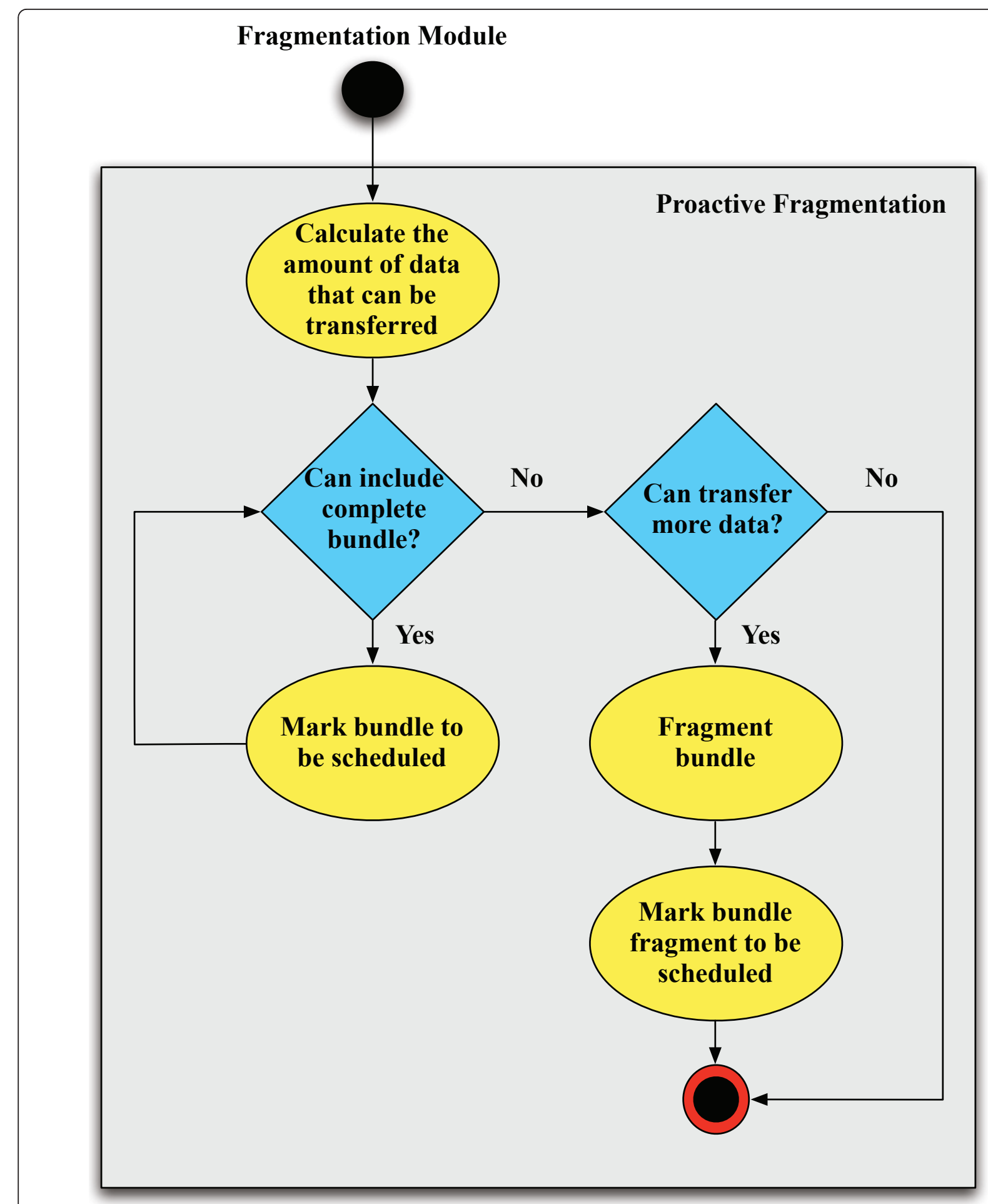

Figure 3 Fragmentation module performing the proactive fragmentation in VDTNs, after concluding the control plane operations

A special case of proactive fragmentation is the source fragmentation. It differs from the above-presented scheme by fragmenting bundles with a given size, at the time of its creation. After the fragmentation at the source nodes, no further fragmentation is done. This means that only fragments will be exchanged between network nodes, on the contrary to what happens in the proactive scheme. Figure 4 illustrates the operations 


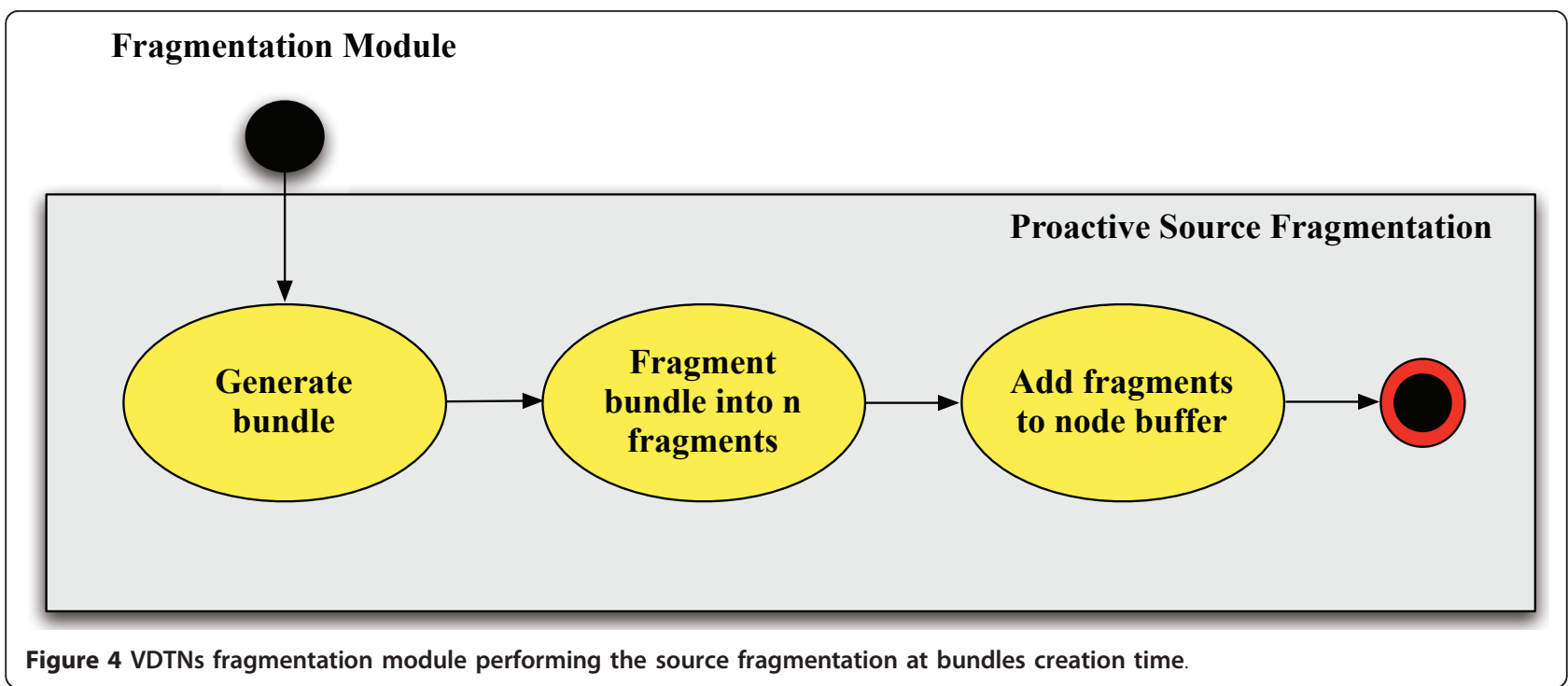

performed by the proactive source fragmentation module during the bundles creation.

\subsection{Reactive fragmentation}

In the reactive fragmentation, two main strategies are considered: reactive and toilet paper. In order to perform the reactive fragmentation approach, at the data plane, nodes have to be able to determine which part of the bundle has been successfully transferred during the data exchange. This process must be performed since the reactive fragmentation is considered when a link failure occurs or when the contact time expires during data exchange, resulting on the transfer of incomplete bundles. The agreement on the part of the bundle that is successfully transferred is done using the following method: the server sends a bundle by blocks, and at the end of each block, an acknowledgment is sent to the client that confirms its reception, sending an acknowledgment to the server. Using this method both sides know exactly which part of the bundle has successfully been transferred. When the communication is broken or interrupted, the receiver creates a fragment with the delivered part of the bundle and the sender creates a fragment with the remaining part of the bundle. Figure 5 illustrates the operations of the fragmentation module for the abovepresented reactive fragmentation scheme.

A special case of reactive fragmentation is the toilet paper approach. The process is identical to the reactive process, but instead of creating a fragment with an arbitrary size, the resulting fragment will have a size defined by the originator.

\section{VDTN@Lab testbed}

The VDTN@Lab is a laboratory testbed that was created to demonstrate, evaluate and validate VDTNs.
Moreover, the VDTN architecture with the different proposed fragmentation approaches was developed and it is shown in Figure 6. It allows the emulation of the VDTN protocols, services and applications. Desktops, laptops, netbooks and robotic cars compose this testbed.

Terminal and relay nodes are emulated using desktops and laptops (iMacs Intel(R) Core(TM) 2 Duo $2.66 \mathrm{GHz}$ +4 GB RAM), while mobile nodes are emulated coupling netbooks (HP Mini Intel(R) Atom $1.66 \mathrm{GHz}+1$ GB RAM) on LEGO MINDSTORMS NXT robots. These LEGO robots are programmed in order to enable different mobility patterns (bus movement or random movement) across roads. All nodes are equipped with Bluetooth and IEEE $802.11 \mathrm{~b} / \mathrm{g}$ devices to allow the separation of control and data planes. Several software modules were deployed on the network nodes to perform the VDTN operations and even several management tools and statistical reports. Figure 7 shows the application user's interface of a mobile node.

For the performance study of the above-presented fragmentation mechanisms, a scenario was set up with three terminal nodes placed at different edges of the laboratory, two relay nodes that are located at roads intersections and four mobile nodes that have a random movement across roads. Deploying a testbed in a laboratory scenario imposes some challenges and constraints, like the required physical space or the representation of the vehicles velocities. Due to space limitations, the number of mobile nodes is limited to four. In addition, these mobile nodes move at different speeds, emulating average velocities about $48,40,36$ and $24 \mathrm{~km} / \mathrm{h}$. These values were obtained taking into account the information gathered from a study performed by real vehicles [26]. In parallel, it is assumed a scale of 1:50 ( $1 \mathrm{~m}$ at the laboratory testbed represents $50 \mathrm{~m}$ in a real scenario). Figure 8 


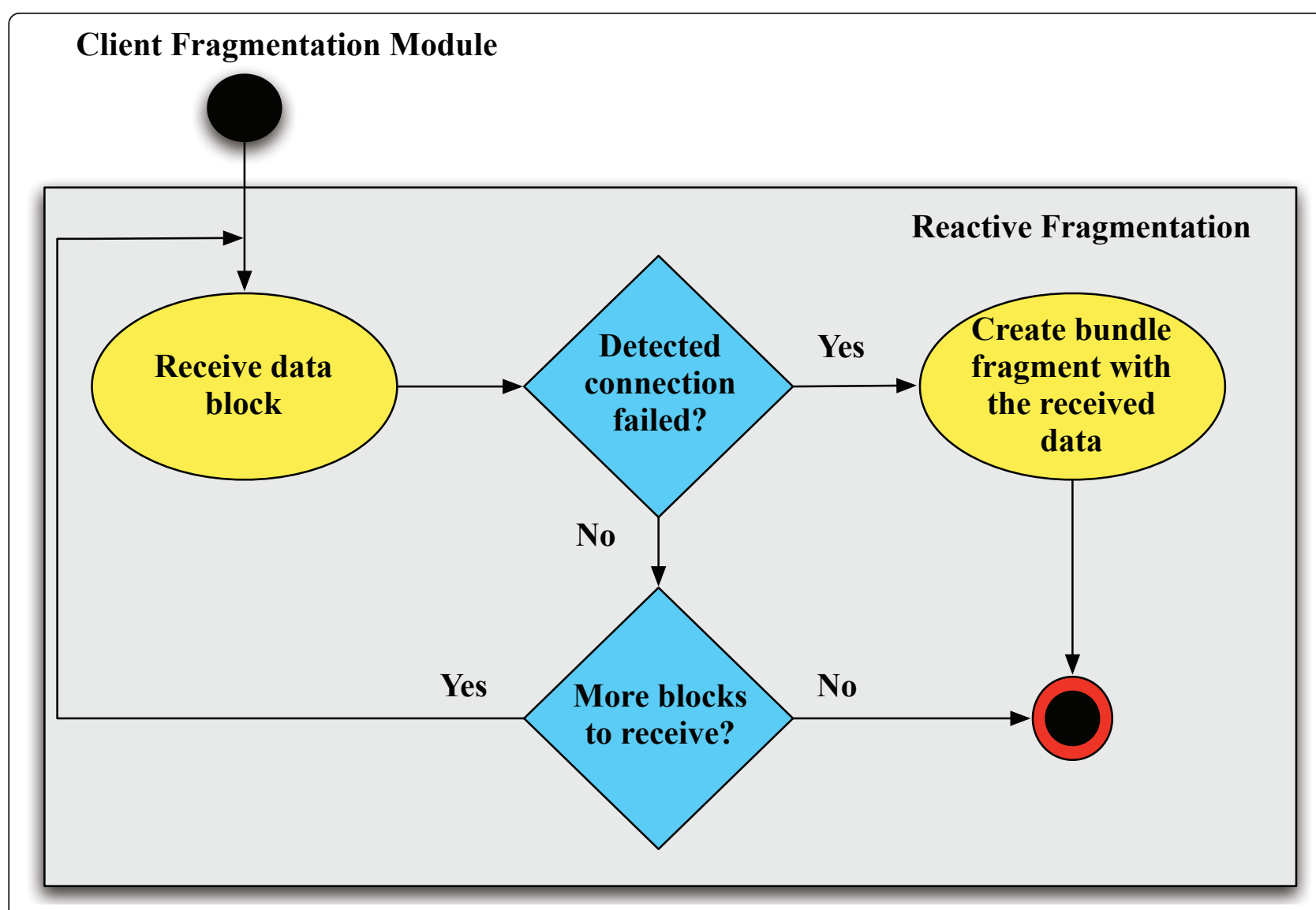

Figure 5 Client fragmentation module operations when a reactive fragmentation is performed.

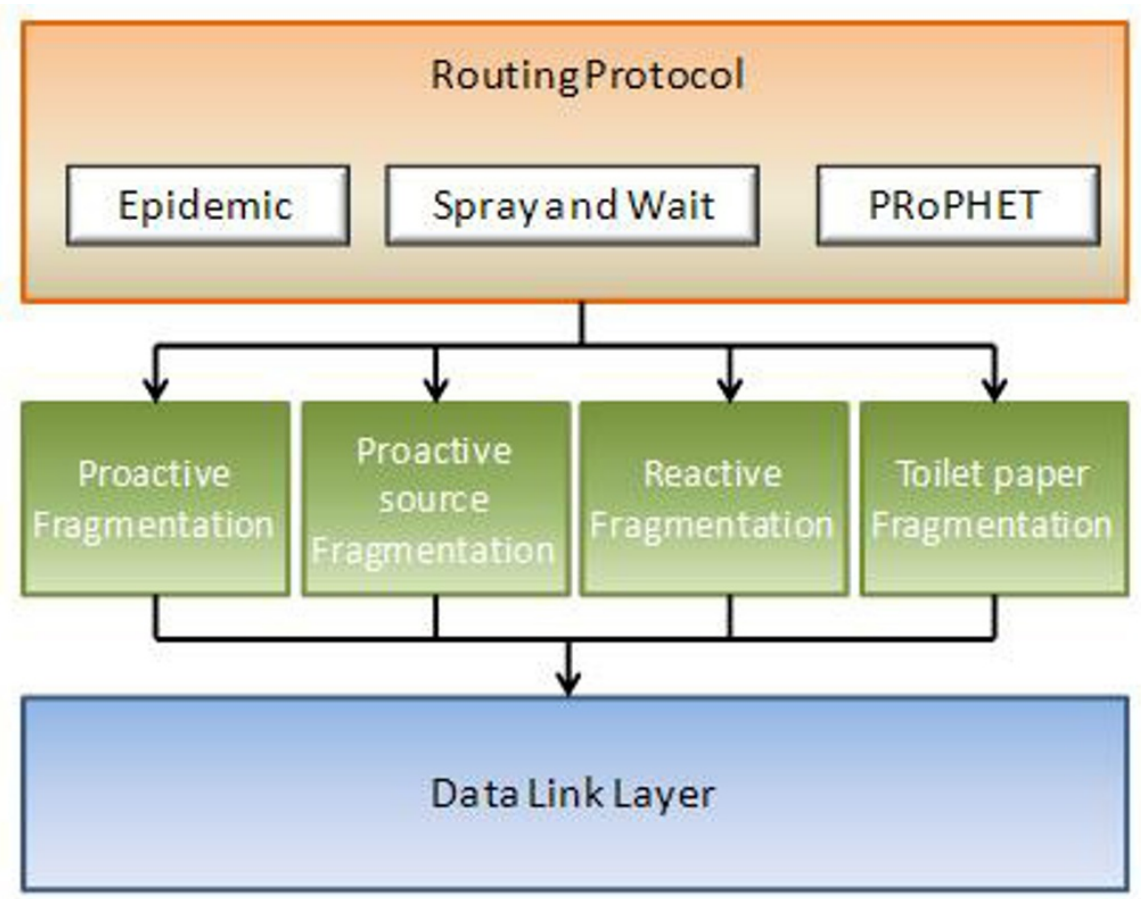

Figure 6 Developed architecture with the proposed fragmentation approaches for VDTN experiments. 


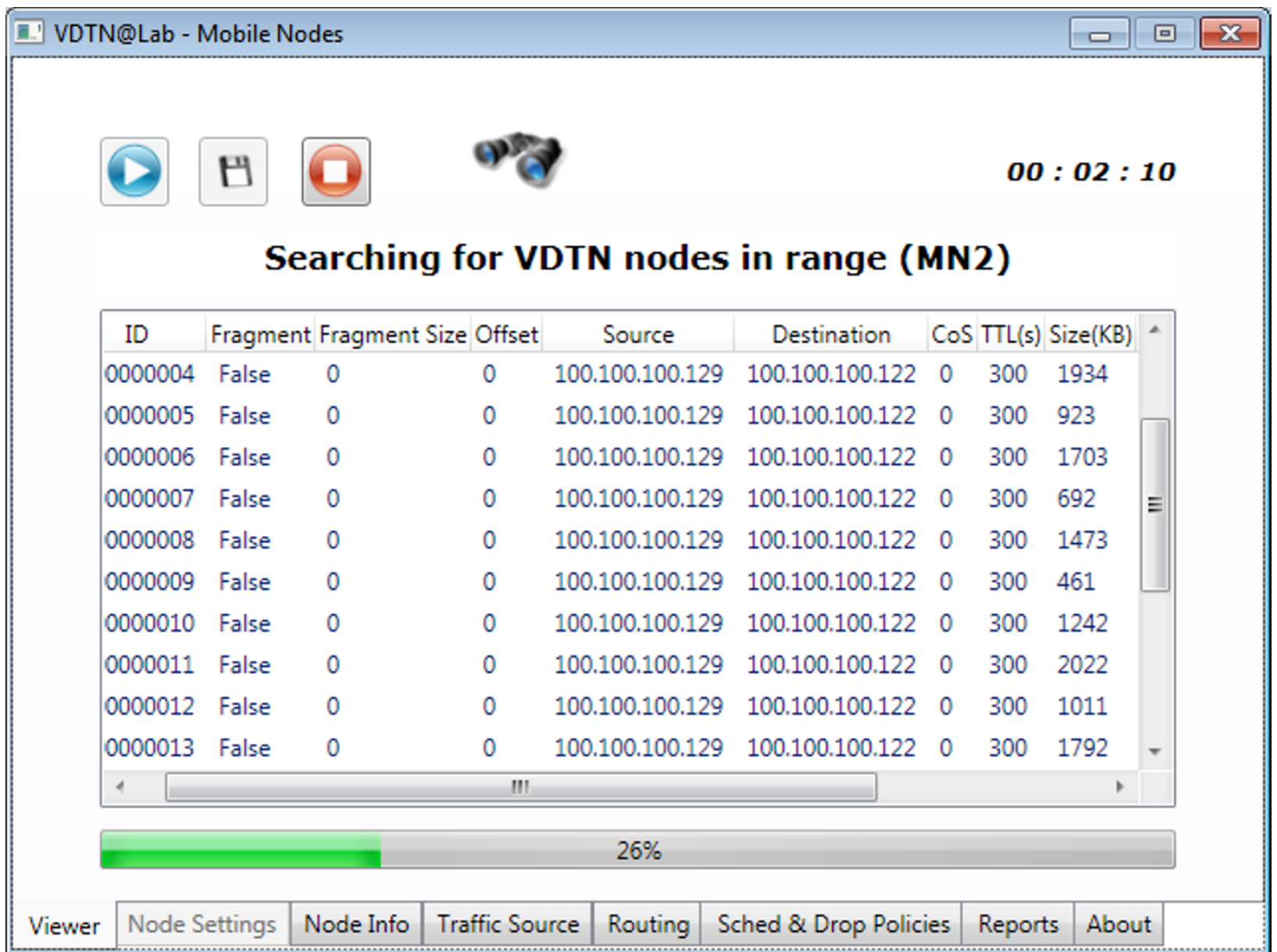

Figure 7 Software interface of a VDTN mobile node.

shows photos of the VDTN@Lab testbed presenting some nodes interactions and behaviours.

The buffer nodes have different capacities according to the functions they perform. Terminal nodes have a buffer with capacity of $50 \mathrm{MB}$, relay nodes have $75 \mathrm{MB}$ and mobile nodes have a buffer with a capacity of about $25 \mathrm{MB}$.

To allow a more accurate study about the impact of fragmentation mechanisms on the performance of VDTNs, a set of configurations should be considered. The software modules generate data bundles every $20 \mathrm{~s}$ with a random destination node. The size of these bundles is uniformly distributed between 256 and 8192 kbytes and its TTL is fixed at $20 \mathrm{~min}$. When buffer congestion occurs or their TTL expires, bundles are dropped, deleting data from buffers according to a FIFO dropping policy. Bundles are scheduled according to a FIFO scheduling policy.

Experiments were conducted considering three different routing protocols, namely, Epidemic [17], binary Spray and Wait [27] and PRoPHET [28]. Epidemic does not require any prior knowledge about the network. In this routing protocol, bundles are replicated to all encountered nodes. Epidemic suffers from the disadvantages of flooding as the node density increases. In an environment with infinite buffer resources and bandwidth, this protocol provides an optimal solution, since it delivers all the bundles that can possibly be delivered in the minimum amount of time.

Spray and Wait limits the number of bundle copies created per bundle in order to control flooding. Bundle copies are initially sprayed (i.e. distributed) to nodes until the number of copies is depleted (in this study it is assumed that this number is 3 ). Two spraying schemes are proposed in [27]. In the source spray scheme, the source node forwards one of the copies to each encountered node until the copy limit is reached. In the binary spray scheme, half of the bundle copies are forwarded to each encountered node. If the destination node is not found during the "spray phase", then at the "wait phase" direct transmission is performed (i.e. the bundle copy left is forwarded only to its destination). 


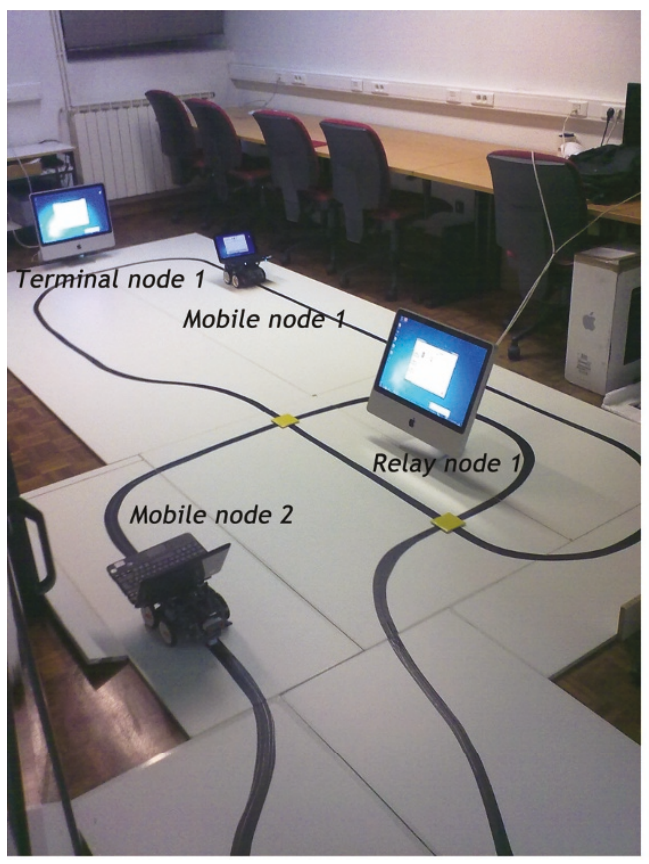

Figure 8 Photos of the VDTN@Lab testbed scenario

PRoPHET is a probabilistic routing protocol. It considers the history of node encounters and transitivity information to calculate a probabilistic metric called delivery predictability. This metric is used to decide whether or not to forward bundles at contact opportunities. A bundle is forwarded to another node if the delivery predictability of the destination of the bundle is higher at that node.

Epidemic, Spray and Wait, and PRoPHET routing protocols have been used as VANET routing protocols in [29-31], respectively.

Performance metrics considered in the conducted experiments are the bundle delivery probability and the bundle average delay. The bundle delivery probability $(B d p)$ is measured as the relation between the number of unique delivered bundles $(U d b)$ and the number of created bundles $(B c)$. Equation 1 shows how this metric is calculated.

$$
B d p=\frac{U d b}{B c}
$$

The bundle average delay $(A d)$ is measured as the average time between bundle creation $(C t)$ and the corresponding delivery $(D t)$. It is calculated according to Equation 2, where $N$ is the number of single delivered bundles.

$$
A d=\frac{\sum_{1}^{N}(D t-C t)}{N}
$$

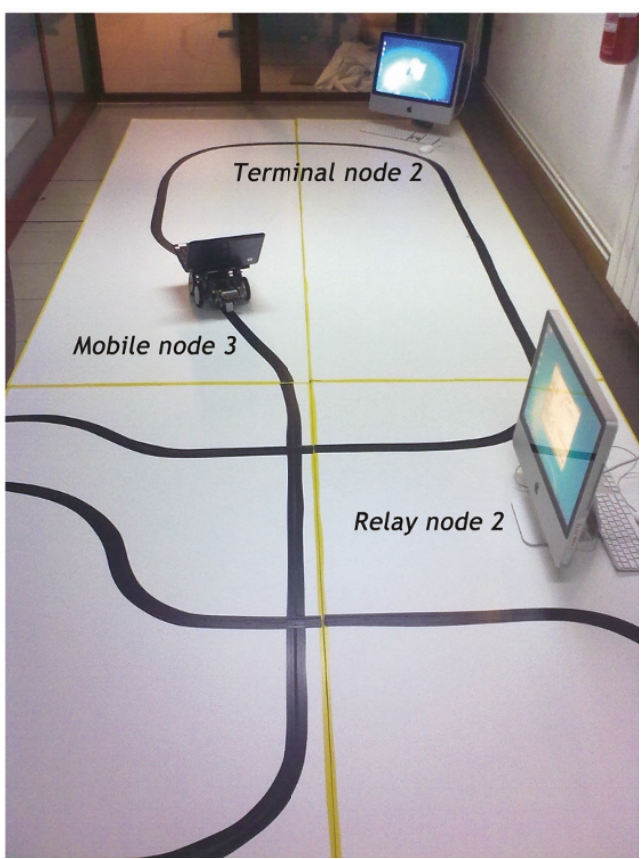

These metrics are recorded for unfragmented bundles and for the four above-presented fragmentation mechanisms: proactive, proactive source (considering three equal size fragment case), reactive and toilet paper (with $128 \mathrm{kB}$ fragments).

\section{Performance analysis}

To analyze the impact of the above-presented fragmentation mechanisms for VDTNs, several experiments were conducted on the VDTN@Lab testbed. Each result represents an average of 30 experiments.

This study starts with the results observed for the different fragmentation mechanisms when the Epidemic routing protocol is considered. As may be seen in Figure 9 , the reactive mechanism contributes to an increase of the bundle delivery probability when compared to the proactive and non-fragmentation approaches. For example, comparing to the non-fragmentation strategy, the reactive mechanism presents gains of $1,3,6,5,17$ and $23 \%$ (for bundles sizes equal to 256, 512, 1024, 2048, 4096 and 8192 kbytes, respectively). The toilet paper approach presents gains of 3, 4, 9, 8, 15 and 22\% when compared to the same non-fragmentation strategy.

Although the proactive mechanism has a very similar performance when compared to the reactive mechanism it performs slightly worse, decreasing the bundle delivery probability in 1, 2, 2, 1, 3 and $4 \%$. The other proactive mechanism, proactive source, always perform worse than the remaining fragmentation approaches, except for the case of bundle size equal to 8192 kbytes, where 


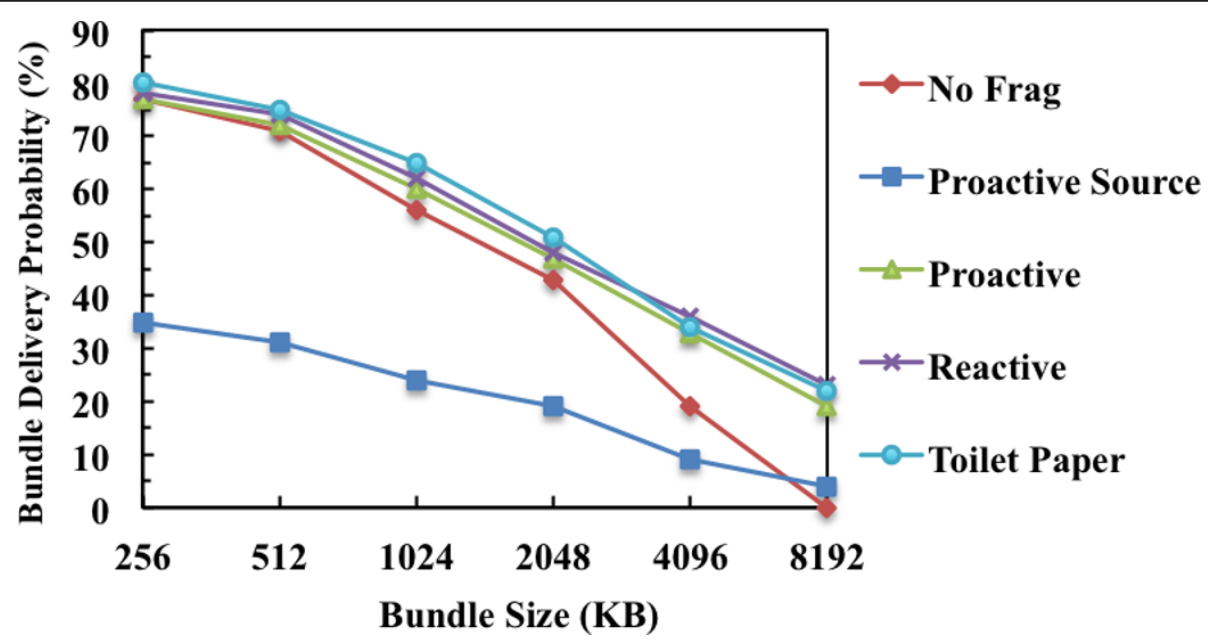

Figure 9 Bundle delivery probability as function of bundle size for the considered fragmentation approaches using Epidemic routing protocol.

the non-fragmentation approach has the worst performance. This occurs because with the proactive source approach, all fragments have to reach the final destination in order to be reassembled into the original bundle. Using Epidemic routing protocol, which makes no control on the bundle replication process, buffer congestion becomes more frequent and most fragments of bundles will be dropped.

The results observed for the bundle average delay are shown in Figure 10. With the introduction of fragmentation mechanisms and the increase of bundle size, bundles tend to be delivered slightly sooner than without fragmentation strategies. However, the latencies are fairly similar not representing a significant gain or loss.
Now the study focuses on the impact of fragmentation mechanisms when the Binary Spray and Wait is considered. Figure 11 shows the obtained results. As may be seen, and like in the previous study, the reactive mechanism perform better than the remaining mechanisms. When compared to the non-fragmentation, reactive mechanism presents gains of 2, 1, 7, 9, 20 and 33\% (for bundles sizes equal to 256, 512, 1024, 2048, 4096 and 8192 kbytes, respectively). The toilet paper approach presents gains of $1,2,8,11,20$ and $30 \%$ when compared with the same non-fragmentation mechanism. The proactive mechanism performs slightly worse, when comparing with the reactive strategies. It increases the bundle delivery probability by about 1, 1, 4, 7, 17 and $29 \%$ when compared to the non-fragmentation approach. As expected,

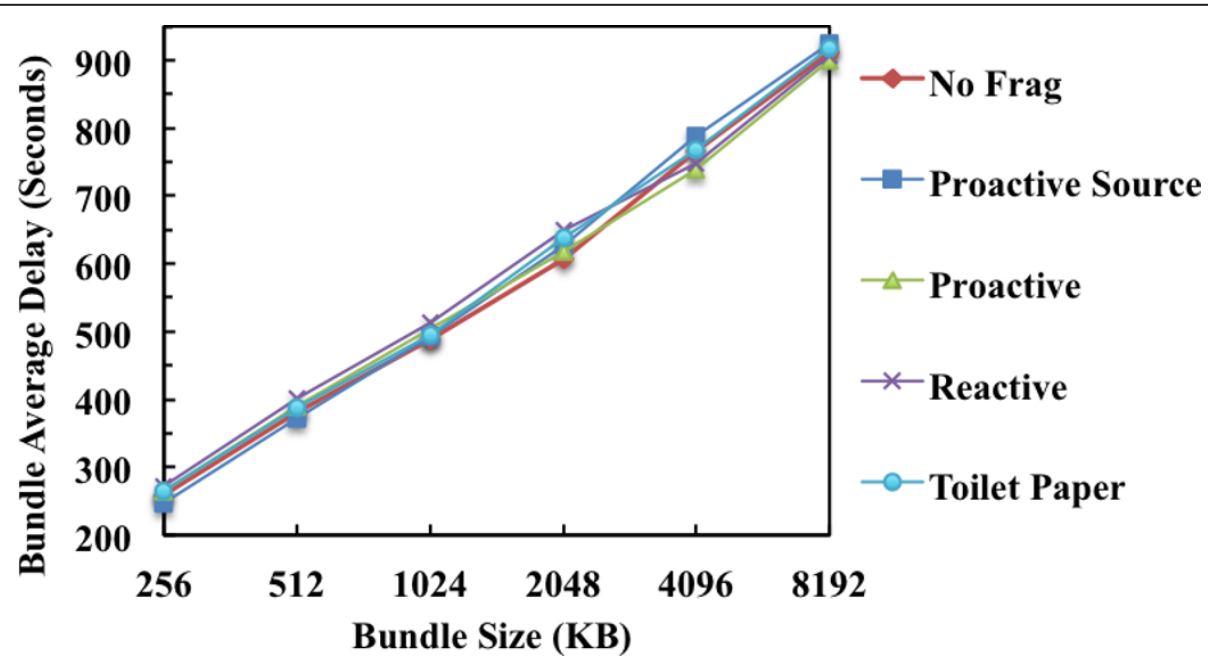

Figure 10 Bundle average delay as function of bundle size for the considered fragmentation approaches using Epidemic routing protocol. 


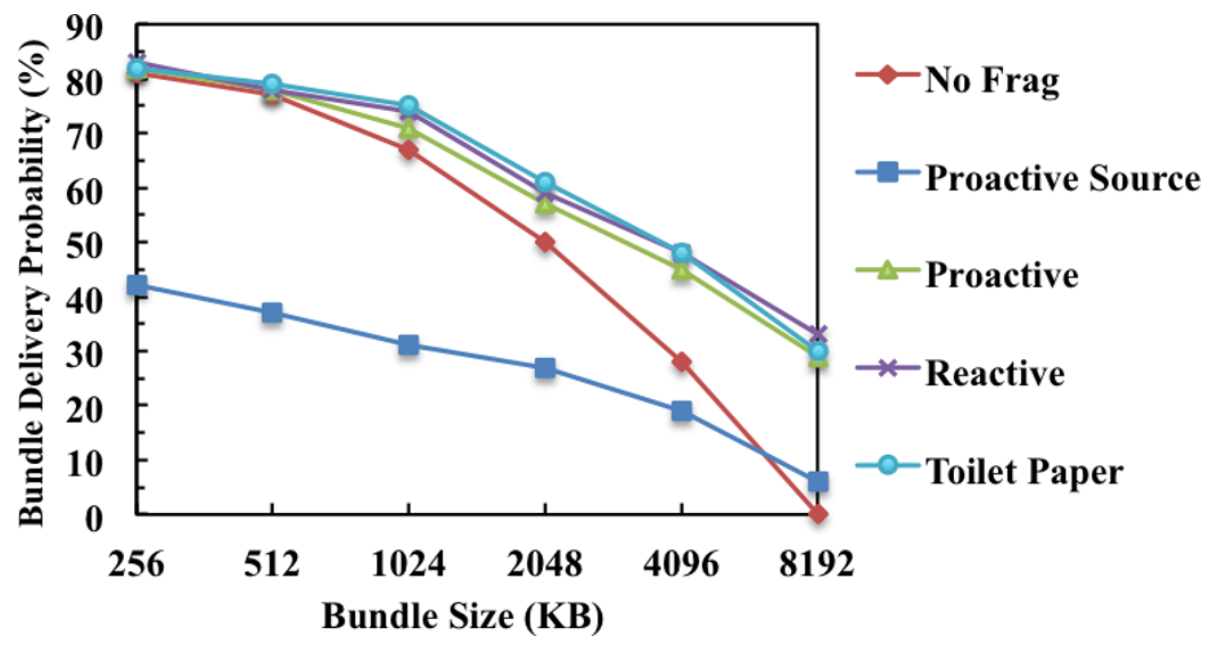

Figure 11 Bundle delivery probability as function of bundle size for the considered fragmentation approaches using Spray and Wait routing protocol.

the proactive source mechanism performs worse than the other fragmentation mechanisms.

The results obtained for the bundle average delay are shown in Figure 12. Like in what happened in the Epidemic study, in this study both reactive and proactive mechanisms tend to deliver bundles slightly sooner than the non-fragmentation mechanism as the bundle size increases. However with the increase of the bundle size, the proactive source mechanism performs worse than the remaining mechanisms.

To conclude this section, the results obtained about the impact of fragmentation mechanisms considering the PRoPHET routing protocol are discussed. As may be seen in Figure 13, and confirming the results obtained in previous studies, the use of both reactive mechanisms contributes to an increase of the bundle delivery probability. The reactive strategy increases the bundle delivery probability by about 2, 8, 6, 13 and $14 \%$ when compared to the non-fragmentation approach (for bundles sizes equal to 512, 1024, 2048 and 4096 kbytes, respectively). Comparatively to the non-fragmentation mechanism, the toilet paper approach increases the bundle delivery probability by about $1,10,11,11$ and $12 \%$.

On the other hand, the proactive mechanism performs slightly worse than the reactive mechanisms. When compared to the reactive mechanism, it decreases the bundle delivery probability by about $1,1,3,2,4$ and $5 \%$. However, when compared to the non-fragmentation mechanism, this mechanism presents gains of 1, 5, 4, 9 and $10 \%$. The proactive source mechanism always

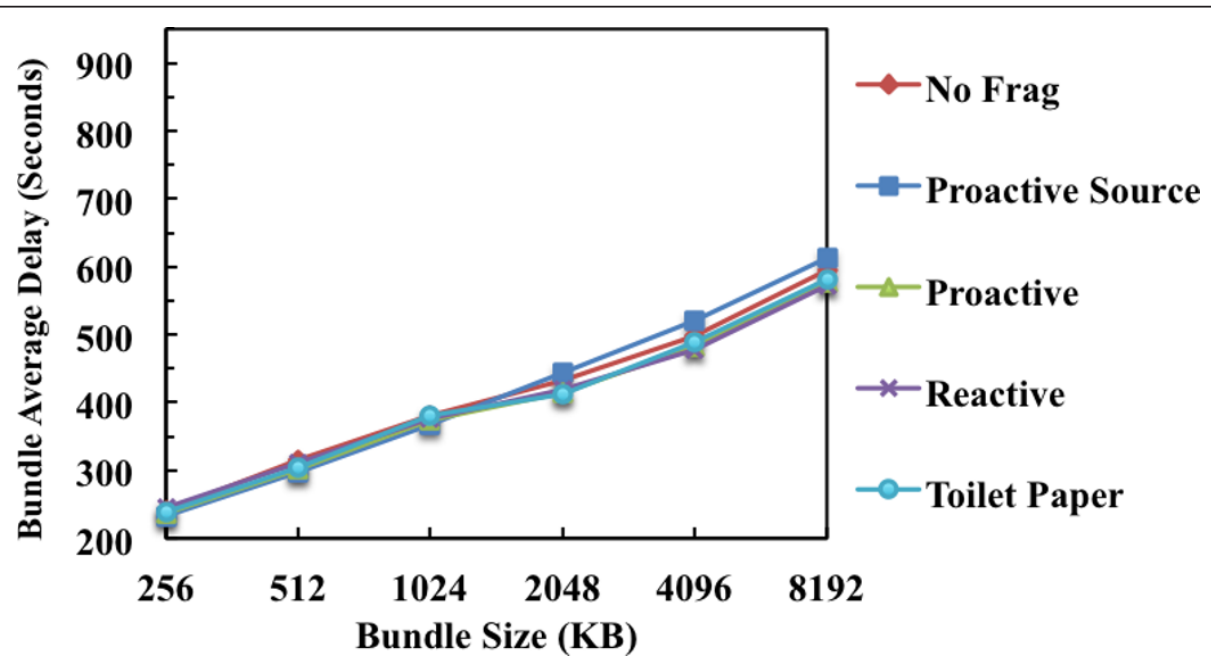

Figure 12 Bundle average delay as function of bundle size for the considered fragmentation approaches using Spray and Wait routing protocol. 


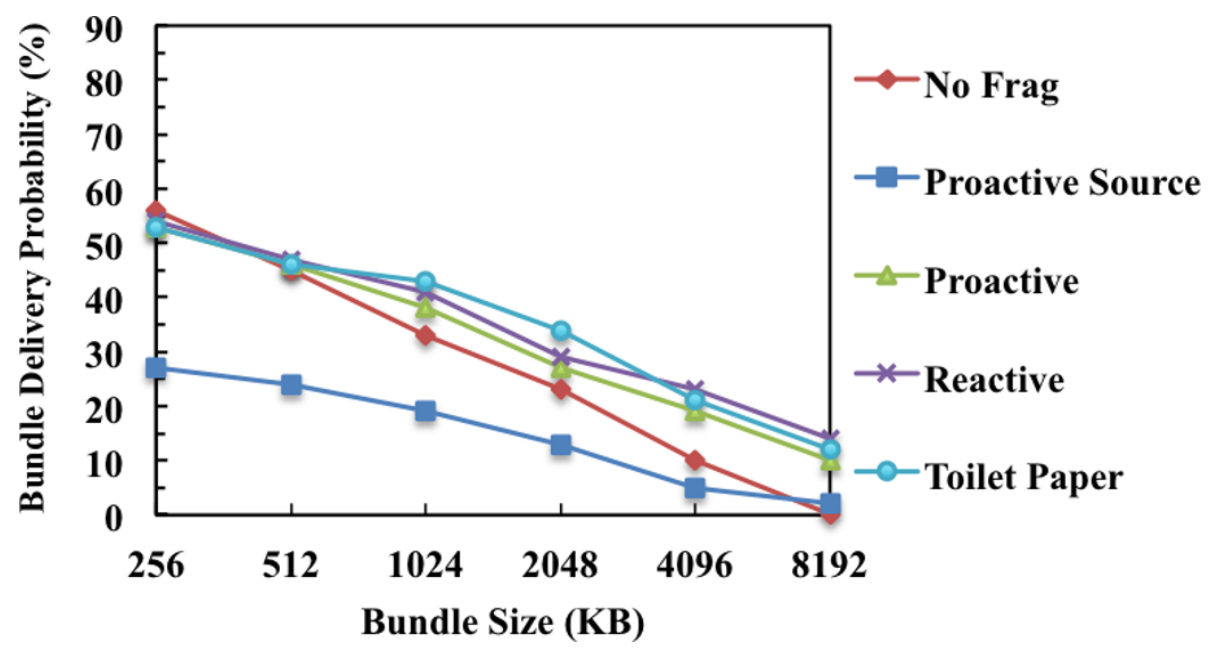

Figure 13 Bundle delivery probability as function of bundle size for the considered fragmentation approaches using PRoPHET routing protocol.

performs worse when compared with the non-fragmentation mechanism except for the case of bundles with 8192 kbytes of size.

With these results it was shown that with the increase of bundles size, the use of fragmentation mechanisms provides a significant gain. This occurs due to the contact durations that are limited on VDTN networks. Increasing bundles size while maintaining contact durations will result in less complete bundles transmitted. Without fragmentation mechanisms, bundles will be lost resulting in a waste of network resources.

Figure 14 presents the results obtained for the same routing protocol but considering the bundle average delay. As may be seen, all the studied fragmentation mechanisms present similar results. However, the proactive source mechanism tends to deliver bundles slightly later as the bundles size increase.

\section{Conclusions and future study}

In this article, several DTN-based fragmentation strategies (proactive, proactive source, reactive and toilet paper) were adapted and deployed on VDTNs. A study considering the impact of these fragmentation mechanisms on the performance of VDTNs was conducted through the VDTN@Lab testbed. Through the experiments, it was observed that reactive fragmentation

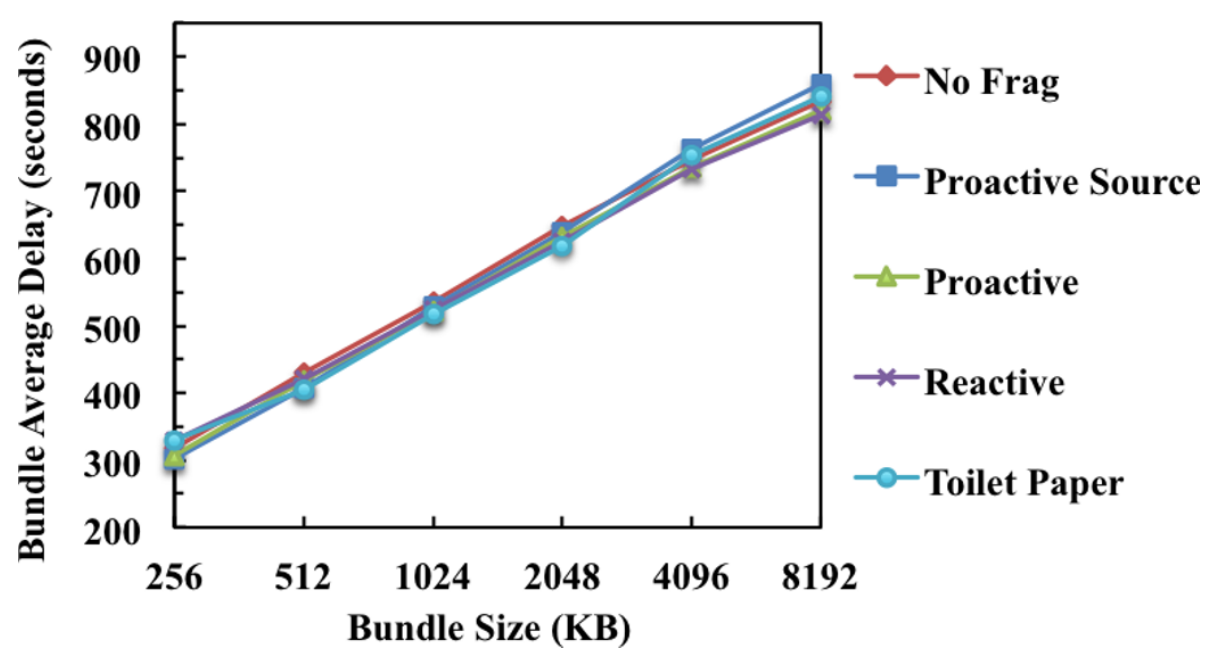

Figure 14 Bundle average delay as function of bundle size for the considered fragmentation approaches using PRoPHET routing protocol. 
appears to be a suitable solution to improve the bundle delivery ratio, while the bundle average delay remains practically the same. The gains are particularly significant for larger bundle sizes.

Experiments were carried out considering the four above-presented fragmentation mechanisms when enforced on three routing protocols: Epidemic, Spray and Wait and PRoPHET. Spray and Wait protocol presents the best results for all the considered fragmentation approaches in terms of bundle delivery probability. This happens since Spray and Wait limits the number of copies in the network. This means that buffer congestion is less likely to occur, or occurs later in each testbed experiment. Because of this, bundles will be dropped mostly due to TTL expiration and not by buffer congestion, allowing more bundles or fragments to reach its final destination.

For all the considered routing protocols, the proactive fragmentation mechanism and both reactive mechanisms improved the bundle delivery ratio when compared to the non-fragmentation case. Only the proactive source mechanism constantly performs worse. The reactive mechanisms perform better due to an improved duplicate detection, leading to a superior buffer utilization that reduces the buffer congestion. However, with the increasing of the bundle size their performance drops. This means that a larger number of fragments are created but not all of them are delivered. In terms of bundle average delay it was shown that different fragmentation mechanisms have a similar performance, when compared to the non-fragmentation mechanisms.

The extension of the laboratory testbed, with the introduction of more routing protocols, such as MaxProp, and more mobility models for mobile nodes are included on the authors' plans for future studies. Networks management, cooperation approaches and a real deployment of VDTNs may also be included for further study.

\section{Acknowledgements}

This study was partially supported by the Instituto de Telecomunicações, Next Generation Networks and Applications Group (NetGNA), Portugal, by the Euro-NF Network of Excellence of the Seventh Framework Programme of EU, in the framework of the Specific Joint Research Project VDTN, and by the INESC-ID multiannual funding through the PIDDAC program funds and National Funding from the FCT - Fundação para a Ciência e a Tecnologia through the PEst-OE/EEI/LA0008/2011 and PTDC/EEA-TEL/099074/2008 (MPSat) Projects.

\section{Author details}

${ }^{1}$ Instituto de Telecomunicações, University of Beira Interior, Covilhã, Portugal ${ }^{2}$ INESC-ID, Instituto Superior Técnico, Technical University of Lisbon, Lisbon, Portugal ${ }^{3}$ Universidad Politécnica de Valencia, Valencia, Spain

\section{Competing interests}

The authors declare that they have no competing interests.
References

1. R Tatchikou, S Biswas, F Dion, Cooperative vehicle collision avoidance using inter-vehicle packet forwarding, Presented at the IEEE Global Telecommunications Conference (IEEE GLOBECOM 2005), St. Louis, MO, USA, 28 November-2 December 2005

2. JS Park, U Lee, SY Oh, M Gerla, DS Lun, Emergency related video streaming in VANET using network coding, in The Third ACM International Workshop on Vehicular Ad Hoc Networks (VANET 2006), Los Angeles, CA, USA, pp. 102-103, September 29, 2006

3. A Buchenscheit, F Schaub, F Kargl, M Weber, A VANET-based emergency vehicle warning system. Presented at the First IEEE Vehicular Networking Conference (IEEE VNC 2009), Tokyo, Japan (28-30 October 2009)

4. M Nekovee, Sensor networks on the road: the promises and challenges of vehicular ad hoc networks and vehicular grids, in Proceedings of the Workshop on Ubiquitous Computing and e-Research, Edinburgh, UK, (May 2005)

5. J Blum, A Eskandarian, L Hoffmman, Challenges of intervehicle ad hoc networks, in IEEE Trans. Intell. Transport. Syst. 5(4), 347-351 (2004). doi:10.1109/TITS.2004.838218

6. S Yousefi, MS Mousavi, M Fathy, Vehicular ad hoc networks (VANETs): challenges and perspectives, in 6th International Conference on ITS Telecommunications (ITST 2006), pp. 761-766 (23 June 2006)

7. H Füßler, M Torrent-Moreno, M Transier, A Festag, H Hartenstein, Thoughts on a protocol architecture for vehicular ad-hoc networks, Presented at the 2nd International Workshop on Intelligent Transportation (WIT 2005), Hamburg, Germany, (15-16 March 2005)

8. V Cerf, S Burleigh, A Hooke, L Torgerson, R Durst, K Scott, K Fall, H Weiss, Delay-tolerant networking architecture, in RFC 4838 http://www.rfc-editor. org/rfc/rfc4838.txt (April 2007).

9. VNGJ Soares, F Farahmand, JJPC Rodrigues, A layered architecture for vehicular delay-tolerant networks, in The Fourteenth IEEE Symposium on Computers and Communications (ISCC 2009), (Sousse, Tunisia), pp. 122-127, 5-8 July 2009

10. JJPC Rodrigues, VNGJ Soares, F Farahmand, Stationary relay nodes deployment on vehicular opportunistic networks, in Mobile Opportunistic Networks: Architectures, Protocols and Applications, ed. by Denko M (CRC Press, Taylor \& Francis Group (hardcover), 2011), pp. 227-243

11. J Postel, Internet Protocol. RFC 791 http://www.ietf.org/rfc/rfc791.txt (September 1981).

12. CA Kent, JC Moguk, Fragmentation considered harmful. SIGCOMM Comput Commun Rev. 25(1), 75-87 (1995). doi:10.1145/205447.205456

13. B-S Kim, Y Fang, TF Wong, Y Kwon, Throughput enhancement through dynamic fragmentation in wireless LANs. IEEE Trans Veh Technol. 54(4), 1415-1425 (2005). doi:10.1109/TVT.2005.851361

14. P Ginzboorg, V Niemi, J Ott, Message Fragmentation in Disruptive Networks, (Nokia Research Center, Technical Report, 2009)

15. M Legner, Map-Based Geographic Forwarding in Vehicular Networks, (Department of Informatic, University of Stuttgart, 2002)

16. Q Li, D Rus, Sending messages to mobile users in disconnected ad-hoc wireless networks, in 6th Annual International Conference on Mobile Computing and Networking, New York, USA, pp. 44-55 (August 2000)

17. A Vahdat, B Becker, Epidemic Routing for Partially-Connected Ad-Hoc Networks, (Duke University, Technical Report, 2000)

18. L Briesemeister, G Hommel, Overcoming fragmentation in mobile ad-hoc networks. J Commun Netw. 2(3), 182-187 (2000)

19. H Liu, H Sheng, Z LV, L Li, C Ma, A cross layer design of fragmentation and priority scheduling in vehicular ad hoc networks, in 7th World Congress on Intelligent Control and Automation (WCICA 2008), pp. 6157-6160 (25-25 June 2008)

20. HP Joshi, Distributed robust geocast: a multicast protocol for inter-vehicle communication, (Master Thesis, North Carolina State University, 2006)

21. A Bachir, A Benslimane, A multicast protocol in ad hoc networks: Intervehicles geocast, in Proceedings of the 57th IEEE Vehicular Technology Conference, Korea, pp. 2456-2460 (April 2003)

22. P Mikko, K Ari, J Ott, Message fragmentation in opportunistic DTNs, in 9th IEEE International Symposium on a World of Wireless, Mobile and Multimedia Networks (WOWMOM 2008), Newport Beach, CA, USA, (23-26 June 2008)

23. S Farrell, S Symington, $H$ Weiss, Delay-tolerant networking security overview. Internet Draft http://tools.ietf.org/html/draft-irtf-dtnrg-secoverview-06 (March 2009). 
24. N Magaia, PR Pereira, A Casaca, J Rodrigues, JA Dias, JN Isento, C CervellóPastor, J Gallego, Bundles fragmentation in vehicular delay-tolerant networks, in 7th Euro-nf conference on next generation internet, Kaiserslautern, Germany (27-29 June 2011)

25. V Soares, J Rodrigues, F Farahmand, M Denko, Exploiting node localization for performance improvement of vehicular delay-tolerant networks, in IEEE International Conference on Communications (ICC 2010), Cape Town, South Africa, (23-27 May 2010)

26. MG Rubinstein, FB Abdesselm, SR Cavalcanti, MEM Campista, RSA Alves, LHMK Costa, MD Amorim, OCMB Duarte, Measuring the capacity of in-car to in-car vehicular networks. IEEE Commun Mag. 47(11), 128-136 (2009)

27. T Spyropoulos, K Psounis, C S Raghavendra, Spray and wait: an efficient routing scheme for intermittently connected mobile networks, in ACM SIGCOMM 2005-Workshop on Delay Tolerant Networking and Related Networks (WDTN-05), (Philadelphia, PA, USA, 22-26 August 2005), pp. 252-259

28. A Lindgren, A Doria, E Davies, S Grasic, Probabilistic routing protocol for intermittently connected networks (2010). Internet Draft http://tools.ietf.org/ html/draft-irtf-dtnrg-prophet-06, (6 July 2010).

29. S Teshima, T Ohta, E Kohno, Y Kakuda, A data transfer scheme using autonomous clustering in VANETs environment, in 10th International Symposium on Autonomous Decentralized Systems (ISADS 2011), (Tokyo, Japan), pp. 477-482 (23-27 March 2011)

30. K Psounis, Efficient Routing for Safety Applications in Vehicular Networks. METRANS Project DTRS98-G0019, Electrical Engineering, University of Southern California, Los Angeles, USA, (2009)

31. X Li, W Shu, M Li, H Huang, Wu Min-You, DTN routing in vehicular sensor networks, in IEEE Global Telecommunications Conference (IEEE GLOBECOM 2008), (New Orleans, USA), pp. 1-5 (30 November-4 December 2008)

doi:10.1186/1687-1499-2011-195

Cite this article as: Dias et al.: Performance assessment of

fragmentation mechanisms for vehicular delay-tolerant networks.

EURASIP Journal on Wireless Communications and Networking 2011

2011:195.

\section{Submit your manuscript to a SpringerOpen ${ }^{\circ}$ journal and benefit from:}

- Convenient online submission

- Rigorous peer review

- Immediate publication on acceptance

- Open access: articles freely available online

- High visibility within the field

- Retaining the copyright to your article

Submit your next manuscript at $\gg$ springeropen.com 